\title{
Comment for the evolving story of overlapping surgery.
}

\author{
Jie Zhang, Zhong Jia ${ }^{1,2 *}$, Chao-Jun Kong ${ }^{1,2}$
}

${ }^{1}$ Department of Hepatopancreatobililiary Surgery, Hangzhou First People's Hospital, Nanjing Medical University Affiliated Hangzhou Hospital, PR China

${ }^{2}$ Department of Hepatopancreatobililiary Surgery, Zhejiang Chinese Medicine University Fourth Affiliated Clinical Hospital, PR China

Accepted on October 31, 2017

\section{Comment}

The evolving story of overlapping surgery described by Michelle et al. [1] indeedly unveiled the tip of the iceberg mainly due to its popularity and rich return. But it also may embrace problematic risks in advertent harm. At first glance, thanks to this instructive and educational analysis on overlapping surgery, many viewpoints are worthy to be learned.

On second look, some issues still let readers confusing. For instance, who on earth often performs overlapping surgery? Why?

As a matter of fact, the primary surgeons are always "experienced master" or "skillful craftman", their technique-art on critical part of surgery will be favorable to promise "safety and efficiency" of patients when and if next surgery is immediate one. But if not immediate, the frequency of overlapping surgery should be restricted not only to ensure quality of surgery but also to benefit professional promotion for inferior trainees. In addition, conflict of interests may be exposed to public when patient ultimately suffers from surgery-related complications or even fatal consequence. The key to resolve the dilemma is to identify a detailed rule from the beginning of overlapping surgery that the primary surgeon must take charge.

We believe overlapping surgery is reasonable as it allows making most use of flow of higher technology-art in good order. And it shines when it comes to rule of law.

\section{References}

1. Mello MM, Livingston EH. "The evolving story of overlapping surgery." JAMA 2017.

\section{*Correspondence to}

Zhong Jia

Department of Hepatopancreatobililiary Surgery

Hangzhou First People's Hospital

Nanjing Medical University Affiliated Hangzhou Hospital \& Zhejiang Chinese Medicine University Fourth Affiliated Clinical Hospital

PR China 